\title{
Everything You Always Wanted to Know about Sex ... in Flies
}

\author{
M.N. Arbeitman ${ }^{\text {a }} \quad$ A. Kopp ${ }^{\text {b }}$ M.L. Siegal ${ }^{c}$ M. Van Doren ${ }^{d}$ \\ aSection of Molecular and Computational Biology, Department of Biological Sciences, University of Southern \\ California, Los Angeles, Calif., ${ }^{b}$ Department of Evolution and Ecology, University of California, Davis, Calif., \\ 'Center for Genomics and Systems Biology, Department of Biology, New York University, New York, N.Y., \\ ${ }^{\mathrm{d}}$ Department of Biology, Johns Hopkins University, Baltimore, Md., USA
}

\section{Key Words}

Drosophila - Gene regulation - Genome evolution •

microRNA - Reproductive physiology - Sex chromosomes •

Sex determination $\cdot$ Sexual dimorphism

\begin{abstract}
'Everything you always wanted to know about sex' is a workshop organized as part of the annual Drosophila Research Conference of the Genetics Society of America. This workshop provides an intellectual venue for interaction among research groups that study sexual dimorphism from the molecular, evolutionary, genomic, and behavioral perspectives. The speakers summarize the key ideas behind their research for people working in other fields, outline unsolved questions, and offer their opinions about future directions. The 2010 workshop highlighted the power of the Drosophila model for understanding sexual dimorphism at levels ranging from cell biology and gene regulation to population genetics and genome evolution, and demonstrated the importance of cross-disciplinary interactions in the study of sex. In this respect, Drosophila sets a good example for research in other organisms, including humans and their mammalian relatives.

Copyright $\odot 2010$ S. Karger AG, Basel
\end{abstract}

Sexual dimorphism arises as an inevitable consequence of evolutionary forces acting on sexually reproducing organisms. Male-female compatibility is clearly essential for successful reproduction. However, male and female reproductive interests do not always agree, giving rise to various forms of sexual selection and sexual conflict. Same-sex (usually male) competition for the reproductive output of the other sex (usually female) leads to the origin and diversification of traits that enable efficient mating and fertilization. This drive may elicit arms' races among male genotypes, as well as between male 'offensive' and female 'defensive' traits. Both processes lead to the evolution of sex-biased, and sometimes sex-limited, phenotypes. When males and females do share the same traits, they often have different fitness optima for these traits. In these cases, alleles that increase fitness in males can decrease it in females, and vice versa, leading to pervasive genetic conflicts.

Both evolutionary and developmental-genetic considerations suggest that a large fraction of the genome should be regulated and/or function in a sex-specific manner. But many questions remain unanswered. What are the genes that mediate sexual selection and sexual conflict? How are these genes regulated, and what are their mo-

\section{KARGER}

Fax +4161306 1234

E-Mail karger@karger.ch

www.karger.com
(C) 2010 S. Karger AG, Basel

$1661-5425 / 10 / 0046-0315 \$ 26.00 / 0$

Accessible online at:

www.karger.com/sxd
Artyom Kopp

Department of Evolution and Ecology, University of California, Davis

One Shields Ave. No. 1, Davis, CA 95616 (USA)

Tel. +1 530752 8328/8657, Fax +1 5307529014

E-Mail akopp@ucdavis.edu 
lecular functions? How do sex-determining mechanisms modify development to produce male- or female-specific morphological, physiological, and behavioral traits? How do male-female interactions occur at the molecular and neurobiological levels? How do new sex-specific phenotypes evolve, and how are the old ones lost? To what extent is genome architecture, especially the sex chromosomes, influenced by the consequences of sexual reproduction? Answering these questions requires interdisciplinary research that pools ideas and technical approaches from both mechanistic and comparative areas of biology.

For the last 2 years, the Drosophila Research Conference organized by the Genetics Society of America (commonly known as the Fly Meeting) included a workshop devoted to the molecular mechanisms and evolution of sexual dimorphism. The annual fly meetings are united by the model organism rather than subject, and range over all areas of Drosophila biology from biochemistry and microbial pathogenesis to neurobiology and population genetics. As a result of this diversity, the fly meetings offer many opportunities for discussions among scientists who think about very different questions yet all speak a common Drosophila language. The goal of the new workshop, entitled 'Everything you always wanted to know about sex', was to provide an intellectual venue for interaction among research groups that study sexual dimorphism from the molecular, evolutionary, genomic, and behavioral perspectives. The speakers summarized the key ideas behind their research for people working in other fields, outlined unsolved questions, and offered their opinions about future directions and cross-disciplinary connections.

In Drosophila, sexual differentiation of most somatic tissues is controlled by an alternative splicing cascade that culminates in the production of sex-specific transcription factors encoded by the doublesex $(d s x)$ gene [Baker and Ridge, 1980; McKeown, 1992]. The male-specific isoform (DsxM) promotes the development of malespecific structures and represses female-specific traits, whereas the female-specific isoform (DsxF) promotes female-specific and represses male-specific traits [Coschigano and Wensink, 1993; Jursnich and Burtis, 1993; Li and Baker, 1998; Waterbury et al., 1999]. The DsxM and DsxF proteins share a common N-terminal DNA-binding domain, but have different $\mathrm{C}$-terminal domains that have different effects on target gene expression [Burtis et al., 1991; Erdman and Burtis, 1993].

The splicing pathway reads out the primary sex determination signal independently in each cell, making it easy to generate mosaic flies composed of a mixture of male and female tissues. Because of this well-known feature, traditional models of sexual differentiation in flies assumed that $d s x$ acted ubiquitously, and that the development of sex-specific traits in some tissues but not others was due to the joint regulation of downstream target genes by the global sex determination pathway and the locally acting spatial patterning genes. However, as Mark Van Doren (Department of Biology, Johns Hopkins University) reviewed at the workshop, recent discoveries from his group and others demonstrate that many cells express neither the male nor the female isoform of $d s x$. Instead, $d s x$ expression is restricted to relatively small populations of cells in the CNS and somatic gonad [Lee et al., 2002; Hempel and Oliver, 2007; Kimura et al., 2008; Sanders and Arbeitman, 2008; Rideout et al., 2010]. Work from the Van Doren group also demonstrates that germline and somatic gonad cells that do not express $d s x$ can nevertheless develop in sexually dimorphic ways. Sexspecific differentiation of these cells is driven by signals emanating from their $d s x$-expressing neighbors. The signaling mechanisms that mediate nonautonomous sexual differentiation include the Jak/Stat, Wnt, FGF, and other signaling pathways. Thus, Drosophila may not be as different from other animals in its sexual development as once thought. Spatially restricted deployment of Dsx-related transcription factors plays a key role in the development of sexually dimorphic tissues in nematodes [Mason et al., 2008] and the vertebrate gonads [Kim et al., 2007], and of course sex-specific development of most somatic tissues in vertebrates is controlled nonautonomously [Koopman, 2001].

Bruce Baker (Janelia Farm Campus, Howard Hughes Medical Institute) expanded on the previous findings by characterizing the spatial pattern of $d s x$ expression in greater detail [Robinett et al., 2010]. The Baker group used a transgenic approach based on homologous recombination to replace the coding sequence of $d s x$ with the coding sequence of the yeast GAL4 transcription factor. This approach places GAL4 under the control of $d s x$ cisregulatory elements in their native chromatin context. The resulting GAL4 reporter faithfully reproduces $d s x$ expression, which can now be examined by crossing the $d s x$-GAL4 strain to flies carrying a UAS-GFP or other GAL4-responsive transgenes. Using this approach, Robinett and her colleagues have shown that $d s x$ is expressed in precisely defined sets of CNS neurons, in many sensory organs, in the epithelial cells that produce sex-specific morphological structures, as well as in some internal tissues that lack overt sexual dimorphism [Robinett et al., 
2010]. Spatial and temporal control of $d s x$ at the transcriptional level can now be seen as a general feature of Drosophila development.

These results radically change our understanding of how sexual differentiation is controlled in Drosophila and, by implication, in other insects. Most cells lack the molecular machinery for interpreting their sex and either need to be instructed by other cells or, more often, remain ignorant of whether they belong to a male or a female. As expressed by Van Doren, sexual identity is revealed on a 'need to know' basis only to those cells that must undergo sex-specific differentiation. Given the ability of $d s x$ to regulate many target genes, including those encoding long-distance signaling molecules, precise transcriptional regulation of $d s x$ may be an essential safety feature. Indeed, ectopic $d s x$ expression can cause developmental abnormalities [Jursnich and Burtis, 1993]. Elucidating the molecular mechanisms that confine $d s x$ transcription to specific tissues and cell types will be essential for understanding the specification of sexually dimorphic structures, behaviors, and physiology.

The spatial regulation of $d s x$ also raises intriguing questions about the evolution of sexually dimorphic traits. Almost every animal lineage has unique sex-specific (often, but not always, male-limited) structures. In Diptera, different families and genera have evolved a variety of sex-specific structures and modifications on virtually every body part, sometimes of truly bizarre proportions, as in the stalk-eyed diopsids and antler-bearing tephritids [Sivinski, 1997]. At the same time, phylogenetic analysis shows that the loss of sex-specific characters occurs at roughly the same rate as the origin of new ones [Wiens, 2001]. Is it possible that the evolutionary gain and loss of sex-specific traits is caused by the origin and loss of tissue-specific $d s x$ expression domains? The answer to this question is unknown but is well worth seeking.

Sexual dimorphism is often a patchwork affair - most sex-specific traits are produced not by the organism as a whole, but by individual tissues and cell types. This implies that most genes responsible for sexual differentiation are regulated sex-specifically only in particular cell types, and may be expressed monomorphically in other tissues. Although more than half of all genes show sexbiased expression in animals as different as Drosophila and vertebrates [Yang et al., 2006; Ellegren and Parsch, 2007], most differences in expression profiles between male and female flies are contributed by the germline [Parisi et al., 2004]. In contrast, only $1-2 \%$ of genes show sex-specific expression in any particular non-gonadal tissue [Arbeitman et al., 2004; Barmina et al., 2005; Kopp et al., 2008; Lebo et al., 2009]. However, these genes show little overlap between different tissues, suggesting that many genes are expressed sex-specifically in at least one somatic cell type. This prediction was confirmed by Richard Meisel (Department of Molecular Biology and Genetics, Cornell University), who used microarray experiments to show that distinct yet overlapping sets of genes are expressed sex-specifically in the head, thorax, and abdomen of D. melanogaster and its distant relative D. willistoni. He further showed that, among genes with male-biased expression, those expressed in many tissues tend to evolve more slowly than those expressed tissuespecifically.

In a parallel approach, Delphine Fagegaltier (Cold Spring Harbor Laboratory) examined genome-wide microRNA (miRNA) expression profiles at different developmental stages and in different adult tissues. Similar to the protein-coding genes, Fagegaltier's work demonstrates that different sets of miRNAs show sex-biased expression in different cells. Some differences are found in every tissue examined, but the gonads are, as expected, the most drastically dimorphic. Although a large fraction of miRNAs exhibit sex-specific regulation at some point in development, only a few are expressed sex-specifically in any given tissue. The roles of miRNAs in sexual dimorphism remain to be understood, but it is conceivable that some of the sex biases in the expression of protein-coding genes are due to post-transcriptional regulation by miRNAs. Identification of the downstream targets of the tissue- and sex-specific miRNAs characterized by Fagegaltier may uncover new regulatory mechanisms for generating sexually dimorphic phenotypes.

Although genome-scale approaches are rapidly expanding our understanding of the molecular basis of sexual dimorphism in flies, we still know surprisingly little about the downstream mechanisms controlling the sexually dimorphic differentiation of specific morphological structures. Stephane Noselli (Institute of Developmental Biology and Cancer, CNRS, and University of Nice - Sophia Antipolis) presented recent work from his laboratory on the peculiar development of male genitalia. In Drosophila and other higher dipterans, the external male genitalia undergo a 360-degree rotation during metamorphosis, finishing exactly where they started but winding the ejaculatory duct around the hindgut in the process [Speder et al., 2006]. This rotation always occurs clockwise as viewed from the posterior, representing a genuine left-right asymmetry marker [Coutelis et al., 2008]. Noselli's group has shown that Myosin ID is required to specify this stereotypical direction of rotation. 
In flies that lack Myosin ID in the 8th abdominal segment, which forms the anterior-most part of the genitalia that interfaces with the abdominal body wall, the genitalia rotate counter-clockwise instead. Rotation is otherwise normal, indicating that Myosin ID is required to specify the direction of asymmetry, but not rotation or asymmetry as such. On the other hand, a novel genetic screen in Noselli's laboratory showed the HOX gene $A b$ dominal $B(A b d-B)$ to be necessary for any rotation to take place. Expression of Myosin ID rescues the $A b d-B$ rotation phenotype, suggesting a hierarchy where $A b d-B$ regulates Myosin ID. Future work may identify additional genes involved in genital rotation, and determine how the sex determination pathway establishes their localized activity. Understanding how this strange and apparently pointless developmental process evolved will require comparative research in other Dipteran families.

The most extreme manifestation of sexual dimorphism is reproduction. Much of the flies' adult lives are subordinated to this overriding necessity, leading to complex coevolution of male and female reproductive physiology. In particular, the ejaculate of Drosophila males contains a complex cocktail of proteins produced by accessory glands [Wolfner, 2007]. Drosophila females can store sperm for many days and typically mate with multiple males during their lifetimes, leading to sperm competition among different males. This competition is mediated in part by the accessory gland proteins (Acps) that affect female physiology and behavior in a variety of ways, including inhibiting the propensity to re-mate and stimulating oviposition. Frank Avila (Department of Molecular Biology and Genetics, Cornell University) showed that a particular glycoprotein, Acp36DE, induces conformational changes in the female reproductive tract that allow the sperm mass contributed by the male to progress to the anterior uterus [Avila and Wolfner, 2009]. These changes are required for the storage and subsequent release of sperm, and are consistent with Acp36DE's role in sperm competition [Chapman et al., 2000]. Also consistent with this role, Acp36DE is a recently evolved and rapidly evolving gene [Wagstaff and Begun, 2005], suggesting that its structure is under strong sexual selection.

The competing requirements of male and female reproduction have a powerful influence on genome structure and evolution. Both sexes share largely the same genome, yet the optimal function of many genes can be very different for males and females. Thus, sexual dimorphism can lead to intralocus sexual conflict - a pattern where many mutations are beneficial to males but detri- mental to females, or vice versa [Bonduriansky and Chenoweth, 2009; van Doorn, 2009]. This tug-of-war can maintain genetic variation in natural populations and prevent either males or females from reaching their respective fitness optima. One way in which this conflict can be resolved is through the evolution of sex-specific gene expression. If the same allele can be expressed differently, and thus induce different phenotypes in males and females, the battle of the sexes is averted [Bedhomme and Chippindale, 2007; Cox and Calsbeek, 2009]. Widespread sex-specific gene expression observed in Drosophila and other animals may thus be a signature of past genetic conflicts. Another such signature may be present in the chromosomal location of genes with male-biased or female-biased expression. Since X-linked genes are hemizygous in males, a recessive mutation that favors male fitness at the expense of females may be positively selected until it reaches high enough frequency to produce a noticeable number of homozygous females. In the long term, however, an X-linked allele spends twice as much time in females than in males, suggesting that for X-chromosomal genes the female functional requirements will tend to win over the male. This process may contribute to the 'demasculinization' of the X chromosome - a pattern where the X chromosome has a significantly lower fraction of genes with male-biased expression than expected by chance [Oliver and Parisi, 2004; Ellegren and Parsch, 2007; Sturgill et al., 2007]. The same trend for miRNAs was reported by Delphine Fagegaltier, who found that miRNAs preferentially expressed in testis versus ovaries tend to reside on the X chromosome. Similarly biased chromosomal distribution of sex-specific miRNAs has also been observed in mice and nematodes [Kato et al., 2009; Song et al., 2009].

However, Richard Meisel's work suggests that the demasculinization of X chromosomes may be more nuanced than appears at first sight. Meisel presented his recent finding that in both $D$. melanogaster and D. willistoni, the dearth of male-biased genes on the $\mathrm{X}$ is indeed observed for genes with sex-biased expression in the thorax and abdomen. However, genes with male-biased expression in the head are actually over-represented on the $\mathrm{X}$ chromosome. The reason for this observation is unclear, but it suggests that genes responsible for sex-specific differentiation of different cell types may be subject to different selective pressures.

The only part of the genome where males and females do not have to struggle for influence is the Y chromosome. It has, however, very few genes - only 12 known genes in D. melanogaster. Consistent with evolutionary 
predictions, all of these genes have functions that are limited to male reproduction. Bernardo Carvalho (Departamento de Genética, Universidade Federal do Rio de Janeiro) reported that different Drosophila species differ in the gene contents of their Y chromosomes: some Y-linked genes of $D$. melanogaster are absent in other species, and vice versa. Using phylogenetic analysis, Carvalho demonstrated that Y-linked genes have been gained and lost on many branches of the Drosophila phylogeny, with the gains greatly outnumbering the losses [Koerich et al., 2008]. Importantly, the closest paralogs of all of these genes are located on autosomes, rather than on the $\mathrm{X}$ chromosome. Based on these observations, Carvalho suggested that the Drosophila Y chromosome is not a degenerate homolog of the $\mathrm{X}$, as in mammals, but rather evolved from a supernumerary ('B') chromosome. B chromosomes are selfish genetic elements found in many insect species [Camacho et al., 2000]. Evolution of meiotic pairing between one of these chromosomes and the $\mathrm{X}$ would turn it into a nascent $\mathrm{Y}$ chromosome that would serve as an evolutionary magnet for male-specific genes since $\mathrm{Y}$ linkage is the surest way to avoid intralocus sexual conflict. The Y-chromosomal toolkit would then be assembled gene by gene by transpositional duplications of autosomal genes, as Carvalho's phylogenetic analysis demonstrates. Consistent with this model, the Y chromosome is not even homologous among Drosophila species. Carvalho's work shows that in D. pseudoobscura and its close relatives, the ancestral Drosophila Y chromosome fused with one of the autosomes so that all the genes that used to be male-limited are now present in both sexes. $D$. pseudoobscura does have a Y chromosome that is essential for male fertility, but the genes it carries are dis- tinct from those found on the $\mathrm{Y}$ chromosomes of other species [Carvalho and Clark, 2005]. These discoveries offer a dramatic example of the extent to which sexual dimorphism shapes genome structure, and show that sex chromosome evolution can take very different courses in different animal lineages. As we learn more about sex determination in non-model organisms, the diversity of evolutionary patterns will probably become ever more obvious.

The workshop has highlighted the power of the Drosophila model for understanding sexual dimorphism at levels ranging from cell biology and gene regulation to population genetics and genome evolution. Perhaps most importantly, it demonstrated the importance of crossdisciplinary interactions in the study of sex. Our growing knowledge of the molecular mechanisms of sexual differentiation raises many questions about how and why these mechanisms evolved, and encourages deeper evolutionary inquiries. At the same time, researchers studying the molecular biology of sexual dimorphism benefit from understanding the evolutionary theories that explain the origin of sex-specific traits, pathways, and genomic features. In this respect, we believe the Drosophila model sets a good example for research in other organisms, including humans and their mammalian relatives.

\section{Acknowledgements}

The authors would like to thank all meeting participants for sharing their results and ideas and for their comments on this review, and the organizers of the Drosophila Research Conference for providing an excellent venue for the workshop.

\section{References}

Arbeitman MN, Fleming AA, Siegal ML, Null $\mathrm{BH}$, Baker BS: A genomic analysis of Drosophila somatic sexual differentiation and its regulation. Development 131:2007-2021 (2004).

Avila FW, Wolfner MF: Acp36DE is required for uterine conformational changes in mated Drosophila females. Proc Natl Acad Sci USA 106:15796-15800 (2009).

-Baker BS, Ridge KA: Sex and the single cell. I. On the action of major loci affecting sex determination in Drosophila melanogaster. Genetics 94:383-423 (1980).
Barmina O, Gonzalo M, McIntyre L, Kopp A: Sex- and segment-specific modulation of gene expression profiles in Drosophila. Dev Biol 288:528-544 (2005).

Bedhomme S, Chippindale AK: Irreconcilable differences: when sexual dimorphism fails to resolve sexual conflict, in Fairbairn DJ, Blanckenhorn WU, Székely T (eds): Sex, Size and Gender Roles, pp 185-195 (Oxford University Press 2007).

Bonduriansky R, Chenoweth SF: Intralocus sexual conflict. Trends Ecol Evol 24:280-288 (2009).
Burtis KC, Coschigano KT, Baker BS, Wensink PC: The doublesex proteins of Drosophila melanogaster bind directly to a sex-specific yolk protein gene enhancer. Embo J 10:25772582 (1991).

Camacho JP, Sharbel TF, Beukeboom LW: Bchromosome evolution. Philos Trans R Soc Lond B Biol Sci 355:163-178 (2000).

Carvalho AB, Clark AG: Y chromosome of $D$. pseudoobscura is not homologous to the ancestral Drosophila Y. Science 307:108-110 (2005).

-Chapman T, Neubaum DM, Wolfner MF, Partridge L: The role of male accessory gland protein Acp36DE in sperm competition in Drosophila melanogaster. Proc Biol Sci 267: 1097-1105 (2000). 
Coschigano KT, Wensink PC: Sex-specific transcriptional regulation by the male and female doublesex proteins of Drosophila. Genes Dev 7:42-54 (1993).

- Coutelis JB, Petzoldt AG, Speder P, Suzanne M, Noselli S: Left-right asymmetry in Drosophila. Semin Cell Dev Biol 19:252-262 (2008).

-Cox RM, Calsbeek R: Sexually antagonistic selection, sexual dimorphism, and the resolution of intralocus sexual conflict. Am Nat 173:176-187 (2009).

-Ellegren H, Parsch J: The evolution of sex-biased genes and sex-biased gene expression. Nat Rev Genet 8:689-698 (2007).

Erdman SE, Burtis KC: The Drosophila doublesex proteins share a novel zinc finger related DNA binding domain. Embo J 12:527-535 (1993).

Hempel LU, Oliver B: Sex-specific DoublesexM expression in subsets of Drosophila somatic gonad cells. BMC developmental biology 7: 113 (2007).

-Jursnich VA, Burtis KC: A positive role in differentiation for the male doublesex protein of Drosophila. Dev Biol 155: 235-249 (1993).

Kato M, de Lencastre A, Pincus Z, Slack FJ: Dynamic expression of small non-coding RNAs, including novel microRNAs and piRNAs/21U-RNAs, during Caenorhabditis elegans development. Genome Biol 10:R54 (2009).

Kim S, Bardwell VJ, Zarkower D: Cell type-autonomous and non-autonomous requirements for Dmrt1 in postnatal testis differentiation. Dev Biol 307:314-327 (2007).

- Kimura K, Hachiya T, Koganezawa M, Tazawa T, Yamamoto D: Fruitless and doublesex coordinate to generate male-specific neurons that can initiate courtship. Neuron 59:759769 (2008).
Koerich LB, Wang X, Clark AG, Carvalho AB: Low conservation of gene content in the Drosophila Y chromosome. Nature 456:949-951 (2008).

Koopman P: The genetics and biology of vertebrate sex determination. Cell 105:843-847 (2001).

Kopp A, Barmina O, Hamilton A, Higgins L, McIntyre L, Jones C: Evolution of gene expression in the Drosophila olfactory system. Mol Biol Evol 25:1081-1092 (2008).

Lebo MS, Sanders LE, Sun F, Arbeitman MN: Somatic, germline and sex hierarchy regulated gene expression during Drosophila metamorphosis. BMC Genomics 10:80 (2009).

Lee G, Hall JC, Park JH: Doublesex gene expression in the central nervous system of Drosophila melanogaster. J Neurogenet 16:229248 (2002).

Li H, Baker BS: Hermaphrodite and doublesex function both dependently and independently to control various aspects of sexual differentiation in Drosophila. Development 125:2641-2651 (1998).

Mason DA, Rabinowitz JS, Portman DS: dmd-3, a doublesex-related gene regulated by tra-1, governs sex-specific morphogenesis in C. elegans. Development 135:2373-2382 (2008).

McKeown M: Sex differentiation: the role of alternative splicing. Curr Opin Genet Dev 2: 299-303 (1992).

Oliver B, Parisi M: Battle of the Xs. Bioessays 26: 543-548 (2004).

- Parisi M, Nuttall R, Edwards P, Minor J, Naiman D, et al: A survey of ovary-, testis-, and somabiased gene expression in Drosophila melanogaster adults. Genome Biol 5:R40 (2004).

Rideout EJ, Dornan AJ, Neville MC, Eadie S, Goodwin SF: Control of sexual differentiation and behavior by the doublesex gene in Drosophila melanogaster. Nat Neurosci 13: 458-466 (2010).
Robinett CC, Vaughan AG, Knapp JM, Baker BS: Sex and the single cell. II. There is a time and place for sex. PLoS Biol 8:e1000365 (2010).

-Sanders LE, Arbeitman MN: Doublesex establishes sexual dimorphism in the Drosophila central nervous system in an isoform-dependent manner by directing cell number. Dev Biol 320:378-390 (2008).

Sivinski J: Ornaments in the Diptera. Florida Entomologist 80:142-164 (1997).

-Song R, Ro S, Michaels JD, Park C, McCarrey JR, Yan W: Many X-linked microRNAs escape meiotic sex chromosome inactivation. Nat Genet 41:488-493 (2009).

-Speder P, Adam G, Noselli S: Type ID unconventional myosin controls left-right asymmetry in Drosophila. Nature 440:803-807 (2006).

- Sturgill D, Zhang Y, Parisi M, Oliver B: Demasculinization of $\mathrm{X}$ chromosomes in the Drosophila genus. Nature 450:238-241 (2007).

van Doorn GS: Intralocus sexual conflict. Ann N Y Acad Sci 1168:52-71 (2009).

Wagstaff BJ, Begun DJ: Comparative genomics of accessory gland protein genes in Drosophila melanogaster and D. pseudoobscura. Mol Biol Evol 22:818-832 (2005).

-Waterbury JA, Jackson LL, Schedl P: Analysis of the doublesex female protein in Drosophila melanogaster: role on sexual differentiation and behavior and dependence on intersex. Genetics 152:1653-1667 (1999).

Wiens JJ: Widespread loss of sexually selected traits: how the peacock lost its spots. Trends Ecol Evol 16:517-523 (2001).

Wolfner MF: 'S.P.E.R.M.' (seminal proteins (are) essential reproductive modulators): the view from Drosophila. Society of Reproduction and Fertility supplement 65:183-199 (2007).

-Yang X, Schadt EE, Wang S, Wang H, Arnold AP, et al: Tissue-specific expression and regulation of sexually dimorphic genes in mice. Genome Res 16:995-1004 (2006). 
